\title{
EIGENVALUES OF PRODUCTS OF UNITARY MATRICES AND QUANTUM SCHUBERT CALCULUS
}

\author{
S. Agnihotri And C. Woodward
}

\begin{abstract}
We describe the inequalities on the possible eigenvalues of products of unitary matrices in terms of quantum Schubert calculus. Related problems are the existence of flat connections on the punctured two-sphere with prescribed holonomies, and the decomposition of fusion product of representations of $S U(n)$, in the large level limit.

In the second part of the paper we investigate how various aspects of the problem (symmetry, factorization) relate to properties of the Gromov-Witten invariants.
\end{abstract}

\section{Introduction}

Beginning with Weyl [32], many mathematicians have been interested in the following question: given the eigenvalues of two Hermitian matrices, what are the possible eigenvalues of their sum? In a recent preprint [18], Klyachko observes that a solution to this problem is given by an application of Mumford's criterion in geometric invariant theory. The eigenvalue inequalities are derived from products in Schubert calculus. In particular, Weyl's inequalities correspond to Schubert calculus in projective space. The necessity of these conditions is due to Helmke and Rosenthal [14].

One of the fascinating points about the above problem are several equivalent formulations noted by Klyachko. For instance, the problem is related to the following question in representation theory: Given a collection of irreducible representations of $S U(n)$, which irreducibles appear in the tensor product? A second equivalent problem involves toric vector bundles over the complex projective plane.

In this paper we investigate the corresponding problem for products of unitary matrices. This question also has a relationship with a representation-theoretic problem, that of the decomposition of the fusion product of representations. The solution to the multiplicative problem is also derived from geometric invariant theory, namely from the Mehta-Seshadri theory of parabolic bundles over the projective line. The main result of this paper, Theorem 3.1, shows that the eigenvalue inequalities are derived from products in quantum Schubert calculus. This improves a result of I. Biswas [5], who gave the first description of these inequalities. A similar result has been obtained independently by P. Belkale [2].

Received December 9, 1997. 
The proof is an application of the Mehta-Seshadri theorem. A set of unitary matrices $A_{1}, \ldots, A_{l}$ such that each $A_{i}$ lies in a conjugacy class $\mathcal{C}_{i}$ and such that their product is the identity is equivalent to a unitary representation of the fundamental group of the $l$ times punctured sphere, with each generator $\gamma_{i}$ being mapped to the conjugacy class $\mathcal{C}_{i}$. By the Mehta-Seshadri theorem such a representation exists if and only if there exists a semi-stable parabolic bundle on $\mathbb{P}^{1}$ with $l$ parabolic points whose parabolic weights come from the choice of conjugacy classes $\mathcal{C}_{i}$. This last interpretation of the original eigenvalue problem can be related to the Gromov-Witten invariants of the Grassmannian and this is done in Section 5 below.

In Sections 6 and 7 we investigate how factorization and hidden symmetries of these Gromov-Witten invariants relate to the multiplicative eigenvalue problem.

\section{Additive inequalities (after Klyachko and Helmke-Rosenthal)}

Let $\mathfrak{s u}(n)$ denote the Lie algebra of $S U(n)$, and

$$
\mathfrak{t}=\left\{\left(\lambda_{1}, \ldots, \lambda_{n}\right) \in \mathbb{R}^{n} \mid \sum \lambda_{i}=0\right\},
$$

its Cartan subalgebra. Let

$$
\mathfrak{t}_{+}=\left\{\left(\lambda_{1}, \ldots, \lambda_{n}\right) \in \mathfrak{t} \mid \lambda_{i} \geq \lambda_{i+1}, \quad i=1, \ldots, n-1\right\}
$$

be a choice of closed positive Weyl chamber. For any matrix $A \in \mathfrak{s u}(n)$ let

$$
\lambda(A)=\left(\lambda_{1}(A), \lambda_{2}(A), \cdots, \lambda_{n}(A)\right) \in \mathfrak{t}_{+}
$$

be the eigenvalues of the Hermitian matrix $-i A$ in non-increasing order. Let $\Delta(l) \subset\left(\mathfrak{t}_{+}\right)^{l}$ denote the set

$\Delta(l)=\left\{\left(\lambda\left(A_{1}\right), \lambda\left(A_{2}\right), \ldots, \lambda\left(A_{l}\right)\right) \mid A_{1}, \ldots, A_{l} \in \mathfrak{s u}(n), A_{1}+A_{2}+\ldots+A_{l}=0\right\}$.

Define an involution

$$
*: \mathfrak{t}_{+} \cong \mathfrak{t}_{+}, \quad\left(\lambda_{1}, \ldots, \lambda_{n}\right) \mapsto\left(-\lambda_{n}, \ldots,-\lambda_{1}\right) .
$$

For any $A \in \mathfrak{s u}(n)$ the matrix $-A$ has eigenvalues $\lambda(-A)=* \lambda(A)$. The set $\Delta(l)$ is invariant under the map

$$
*^{l}:\left(\mathfrak{t}_{+}\right)^{l} \rightarrow\left(\mathfrak{t}_{+}\right)^{l}, \quad\left(\xi_{1}, \ldots, \xi_{l}\right) \mapsto\left(* \xi_{1}, \ldots, * \xi_{l}\right),
$$

and also under the action of the symmetric group $S_{l}$ on $\left(\mathfrak{t}_{+}\right)^{l}$.

The set $\Delta(l)$ has interesting interpretations in symplectic geometry and representation theory. Consider the cotangent bundle $T^{*} S U(n)^{l-1}$ with the action of $S U(n)^{l}$ given by $S U(n)$ acting diagonally on the left and $S U(n)^{l-1}$ on the right. The moment polytope of this action may be identified with $\Delta(l)$ (see Section 5.) From the convexity theorem for proper moments proved by Sjamaar [30] (see also [20]), it follows that $\Delta(l)$ is a finitely-generated convex polyhedral cone. In particular there are a finite number of inequalities defining $\Delta(l)$ as a subset of the polyhedral cone $\left(\mathfrak{t}_{+}\right)^{l}$. 
The set $\Delta(l)$ may also be described in terms of the tensor product of representations. Let

$$
(, \quad): \mathfrak{s u}(n) \times \mathfrak{s u}(n) \rightarrow \mathbb{R}, \quad(A, B) \mapsto-\operatorname{Tr}(A B),
$$

denote the basic inner product on $\mathfrak{s u}(n)$, which induces an identification $\mathfrak{s u}(n) \cong$ $\mathfrak{s u}(n)^{*}$. Let $\Lambda=\mathbb{Z}^{n} \cap \mathfrak{t}$ denote the integral lattice and $\Lambda^{*} \subset \mathfrak{t}$ its dual, the weight lattice. For each $\lambda \in \Lambda^{*} \cap \mathfrak{t}_{+}$, let $V_{\lambda}$ denote the corresponding irreducible representation of $S U(n)$. We will see in equation (9) that $\Delta(l) \cap \mathbb{Q}^{l}$ is the set of $\left(\lambda^{1}, \ldots, \lambda^{l}\right)$ such that for some $N$ such that $N \lambda^{1}, \ldots, N \lambda^{l} \in \Lambda^{*}$, we have

$$
V_{N \lambda^{1}} \otimes \ldots \otimes V_{N \lambda^{l-1}} \supset V_{N \lambda^{l}}^{*},
$$

that is, $V_{N \lambda^{1}} \otimes \ldots \otimes V_{N \lambda^{l}}$ contains a non-zero invariant vector.

The work of Klyachko and Helmke-Rosenthal gives a necessary and sufficient set of inequalities describing $\Delta(l)$ in terms of Schubert calculus. Let

$$
\mathbb{C}^{n}=F_{n} \supset F_{n-1} \supset \ldots \supset F_{0}=\{0\}
$$

be a complete flag in $\mathbb{C}^{n}, G(r, n)$ the Grassmanian of $r$-planes in $\mathbb{C}^{n}$, and for any subset $I=\left\{i_{1}, \ldots, i_{r}\right\} \subset\{1, \ldots n\}$, let

$$
\sigma_{I}=\left\{W \in G(r, n) \mid \operatorname{dim}\left(W \cap F_{i_{j}}\right) \geq j, j=1, \ldots, r\right\}
$$

denote the corresponding Schubert variety. The Schubert cell $C_{I} \subset \sigma_{I}$ is defined as the complement of all lower-dimensional Schubert varieties contained in $\sigma_{I}$ :

$$
C_{I}=\sigma_{I} \backslash \bigcup_{\sigma_{J} \subset \sigma_{I}} \sigma_{J}
$$

We say that $W$ is in position $I$ with respect to the flag $F_{*}$ if $W \in C_{I}$.

The homology classes $\left[\sigma_{I}\right]$ form a basis of $H_{*}(G(r, n), \mathbb{Z})$. Given two Schubert cycles $\sigma_{I}, \sigma_{J}$, we can expand the intersection product $\left[\sigma_{I}\right] \cap\left[\sigma_{J}\right]$ in terms of this basis. We say $\left[\sigma_{I}\right] \cap\left[\sigma_{J}\right]$ contains $\left[\sigma_{K}\right]$ if $\left[\sigma_{K}\right]$ appears in this expansion with non-zero (and therefore positive) coefficient. Equivalently, let

$$
* K=\left\{n+1-i_{r}, n+1-i_{r-1}, \ldots, n+1-i_{1}\right\},
$$

so that $\left[\sigma_{* K}\right]$ is the Poincare dual of $\left[\sigma_{K}\right]$. Then $\left[\sigma_{I}\right] \cap\left[\sigma_{J}\right]$ contains $\left[\sigma_{K}\right]$ if and only if the intersection of general translates of the Schubert cycles $\sigma_{I}, \sigma_{J}, \sigma_{* K}$ is non-empty and finite.

Theorem 2.1 (Klyachko, resp. Helmke-Rosenthal). A necessary and sufficient (resp. necessary) set of inequalities describing $\Delta(l)$ as a subset of $\left(\mathfrak{t}_{+}\right)^{l}$ are

$$
\sum_{i \in I_{1}} \lambda_{i}\left(A_{1}\right)+\sum_{i \in I_{2}} \lambda_{i}\left(A_{2}\right)+\ldots+\sum_{i \in I_{l}} \lambda_{i}\left(A_{l}\right) \leq 0
$$

where $I_{1}, \ldots, I_{l}$ are subsets of $\{1, \ldots, n\}$ of the same cardinality $r$ such that $\left[\sigma_{I_{1}}\right] \cap \ldots \cap\left[\sigma_{I_{l-1}}\right] \supset\left[\sigma_{* I_{l}}\right]$, and $r$ ranges over all values between 1 and $n-1$. 
Note that the cases $l=1,2$ are trivial: $\Delta(1)=\{0\}$, and $\Delta(1)=\{(\mu, * \mu) \mid \mu \in$ $\left.\mathfrak{t}_{+}\right\}$. Klyachko also claims that these inequalities are independent. ${ }^{1}$

From Theorem 2.1 follows a complete set of inequalities for the possible eigenvalues of a sum of skew-Hermitian matrices. For instance, for $l=3$ one obtains the inequalities

$$
\sum_{i \in I} \lambda_{i}(A)+\sum_{j \in J} \lambda_{j}(B) \leq \sum_{k \in K} \lambda_{k}(A+B)
$$

where $I, J, K \subset\{1, \ldots, n\}$ range over subsets such that $\left[\sigma_{I}\right] \cap\left[\sigma_{J}\right]$ contains $\left[\sigma_{K}\right]$.

Example 2.2. Let $r=1$ so that $G(r, n) \cong \mathbb{P}^{n-1}$ and $I=\{n-i+1\}, J=$ $\{n-j+1\}$. Then $\sigma_{I} \cong \mathbb{P}^{n-i}, \sigma_{J} \cong \mathbb{P}^{n-j}$ so that $\left[\sigma_{I}\right] \cap\left[\sigma_{J}\right] \cong \mathbb{P}^{n-i-j+1}=\sigma_{K}$ where $K=\{n-i-j+2\}$. One obtains

$$
\lambda_{n-i+1}(A)+\lambda_{n-j+1}(B) \leq \lambda_{n-i-j+2}(A+B) .
$$

2.1. Duality. Let $A_{1}, \ldots, A_{l} \in \mathfrak{s u}(n)$. From (2) applied to $-A_{1}, \ldots,-A_{l}$ one obtains

$$
-\sum_{i \in * I_{1}} \lambda_{i}\left(A_{1}\right)-\ldots-\sum_{i \in * I_{l}} \lambda_{i}\left(A_{l}\right) \leq 0
$$

or equivalently, $\sum_{i \in * I_{1}} \lambda_{i}\left(A_{1}\right)+\ldots+\sum_{i \in * I_{l}} \lambda_{i}\left(A_{l}\right) \geq 0$. By the trace condition, (4) is equivalent to

$$
\sum_{i \notin * I_{1}} \lambda_{i}\left(A_{1}\right)+\ldots+\sum_{i \notin * I_{l}} \lambda_{i}\left(A_{l}\right) \leq 0
$$

Let $I_{i}^{c}=\{1, \ldots, n\} \backslash * I_{i}$. Then $\left[\sigma_{I_{i}^{c}}\right]$ is the image of $\left[\sigma_{I_{i}}\right]$ under the isomorphism of homology induced by $G(r, n) \cong G(n-r, n)$ (see page 197 onwards of Griffiths and Harris [10]). Thus the appearance of (4) in (1) corresponds to a product in the Schubert calculus of $G(n-r, n)$.

Example 2.3. The dual equation to (3) is Weyl's 1912 [32] inequality

$$
\lambda_{i}(A)+\lambda_{j}(B) \geq \lambda_{i+j-1}(A+B) .
$$

\footnotetext{
${ }^{1}$ We discovered after the first draft of this paper was circulated that in fact these inequalities are not independent. In the special case $n=4$ and $b=4$, the inequalities with intersection number equal to one form a complete and independent set of inequalities describing $\Delta$. The unique redundant inequality in this case corresponds to the product $\sigma_{\{2,4\}}^{n 4}=2[\mathrm{pt}]$. Fulton observed that this inequality is implied by the inequalities that define the positive chamber. Belkale [2] has proved that for arbitrary $n$ and $b$, the inequalities with intersection number greater than one are redundant. The independence of the remaining inequalities is, at this time, still open.
} 


\section{Multiplicative Inequalities}

Let $\mathfrak{A} \subset \mathfrak{t}_{+}$be the fundamental alcove of $S U(n)$ :

$$
\mathfrak{A}=\left\{\lambda \in \mathfrak{t}_{+} \mid \lambda_{1}-\lambda_{n} \leq 1\right\} .
$$

Let $A \in S U(n)$ be a unitary matrix with determinant 1 . Its eigenvalues may be written

$$
e^{2 \pi i \lambda_{1}(A)}, e^{2 \pi i \lambda_{2}(A)}, \ldots, e^{2 \pi i \lambda_{n}(A)},
$$

where $\lambda(A)=\left(\lambda_{1}(A), \ldots, \lambda_{n}(A)\right) \in \mathfrak{A}$. The map $A \mapsto \lambda(A)$ induces a homeomorphism

$$
\mathfrak{A} \cong S U(n) / \operatorname{Ad}(S U(n)) .
$$

Let $\Delta_{q}(l) \subset \mathfrak{A}^{l}$ ( $q$ for quantum) denote the set

$$
\Delta_{q}(l)=\left\{\left(\lambda\left(A_{1}\right), \ldots, \lambda\left(A_{l}\right)\right) \mid A_{1}, \ldots, A_{l} \in S U(n), A_{1} A_{2} \ldots A_{l}=I\right\} .
$$

As before, $\Delta_{q}(l)$ is invariant under the involution, $*^{l}: \mathfrak{A}^{l} \rightarrow \mathfrak{A}^{l}$, and the action of the symmetric group $S_{l}$ on $\mathfrak{A}^{l}$.

The set $\Delta_{q}(l)$ has an interpretation as a moment polytope. Let $\mathcal{M}$ be the space of flat $S U(n)$-connections on the trivial $S U(n)$ bundle over the $l$-holed twosphere, modulo gauge transformations which are the identity on the boundary (see [24]). The gauge group of the boundary acts on $\mathcal{M}$ in Hamiltonian fashion and the set $\Delta_{q}(l)$ is the moment polytope for this action. By [24, Theorem 3.19], $\Delta_{q}(l)$ is a convex polytope. In fact, an analogous statement holds for arbitrary compact, simply-connected Lie groups. In particular, a finite number of inequalities describe $\Delta_{q}(l)$. In the case $n=2$, these inequalities were given explicitly for $l=3$ in Jeffrey-Weitsman [15] and for arbitrary numbers of marked points in Biswas [6]. A description of the inequalities in the arbitrary rank case was given in [5] but the description given there does not seem to be computable.

There is also an interpretation of $\Delta_{q}(l)$ in terms of fusion product. Let $\circledast_{N}$ denote the fusion product on the Verlinde algebra $R\left(S U(n)_{N}\right)$ of $S U(n)$ at level $N$. Then $\Delta_{q}(l) \cap \mathbb{Q}^{l}$ is the set of $\left(\lambda^{1}, \ldots, \lambda^{l}\right) \in \mathfrak{A} \cap \mathbb{Q}^{l}$ such that for some $N$ such that $N \lambda^{1}, \ldots, N \lambda^{l} \in \Lambda^{*}$, we have

$$
V_{N \lambda^{1}} \circledast_{N} \ldots \circledast_{N} V_{N \lambda^{l-1}} \supset V_{N * \lambda^{l}} .
$$

See Section 8.

3.1. Quantum Schubert calculus. Quantum cohomology is a deformation of the ordinary cohomology ring that was introduced by the physicists Vafa and Witten. Quantum cohomology of the Grassmannian (quantum Schubert calculus) was put on a rigorous footing by Bertram [3]. Recall that the degree of a holomorphic map $\varphi: \mathbb{P}^{1} \rightarrow G(r, n)$ is the homology class $[\varphi] \in H^{2}(G(r, n), \mathbb{Z}) \cong$ $\mathbb{Z}$. Let $p_{1}, \ldots, p_{l}$ be distinct marked points in $\mathbb{P}^{1}$. The quantum intersection product $\star$ on $H_{*}(G(r, n), \mathbb{C}) \otimes \mathbb{C}[q]$ is defined by

$$
\left[\sigma_{I_{1}}\right] \star \ldots \star\left[\sigma_{I_{l}}\right]=\sum_{J}\left\langle\left[\sigma_{I_{1}}\right], \ldots,\left[\sigma_{I_{l}}\right],\left[\sigma_{J}\right]\right\rangle_{d}\left[\sigma_{* J}\right] q^{d}
$$


where the Gromov-Witten invariant $\left\langle\left[\sigma_{I_{1}}\right], \ldots,\left[\sigma_{I_{l}}\right],\left[\sigma_{J}\right]\right\rangle_{d}$ is equal to the number of holomorphic maps $\mathbb{P}^{1} \mapsto G(r, n)$ sending $p_{1}, \ldots, p_{l}, p$ to general translates of $\sigma_{I_{1}}, \ldots, \sigma_{I_{l}}, \sigma_{J}$ if this number is finite, and is otherwise zero.

Our main result is the following description of $\Delta_{q}(l)$ :

Theorem 3.1. A necessary and sufficient set of inequalities for $\Delta_{q}(l)$ are given by

$$
\sum_{i \in I_{1}} \lambda_{i}\left(A_{1}\right)+\sum_{i \in I_{2}} \lambda_{i}\left(A_{2}\right)+\ldots+\sum_{i \in I_{l}} \lambda_{i}\left(A_{l}\right) \leq d,
$$

for $\left(I_{1}, \ldots, I_{l}, d\right)$ such that $\left\langle\left[\sigma_{I_{1}}\right] \star \ldots \star\left[\sigma_{I_{l}}\right]\right\rangle_{d} \neq 0$. That is, $\left[\sigma_{I_{1}}\right] \star \ldots \star\left[\sigma_{I_{l-1}}\right] \supset$ $q^{d}\left[\sigma_{* I_{l}}\right]$.

In the last few years several techniques have been developed for computing the coefficients of quantum Schubert calculus. See for instance Bertram, CiocanFontanine, Fulton [4]. Therefore the above theorem makes the question of which inequalities occur computable in practice.

One recovers the inequalities for $\Delta(l)$ from the degree 0 Gromov-Witten invariants. This shows that $\Delta(l)$ is the cone on $\Delta_{q}(l)$ at the 0 -vertex, i.e.

$$
\Delta(l)=\mathbb{R}_{+} \cdot \Delta_{q}(l) .
$$

This may be verified by several alternative methods, e.g. Remark 5.4.

The simplest example of a positive degree inequality is given by the following:

Example 3.2. Let $r=1$ so that $G(r, n)=\mathbb{P}^{n-1}$, and $U, V, W \subset \mathbb{C}^{n}$ be subspaces in general position of dimensions $i, j, n+1-i-j$. There is a unique degree 1 map $\mathbb{P}^{1} \rightarrow \mathbb{P}^{n-1}$ mapping $p_{1}, p_{2}, p_{3}$ to $\mathbb{P}(U), \mathbb{P}(V), \mathbb{P}(W)$ respectively. Together with the degree 0 inequality mentioned before, this gives

$$
\lambda_{i+j-1}(A B) \leq \lambda_{i}(A)+\lambda_{j}(B) \leq \lambda_{i+j}(A B)+1 .
$$

We will see in Section 7 that these inequalities are related by a symmetry of $\Delta_{q}(l)$.

\section{Moduli of flags and Mumford's criterion}

As a warm-up we review some of the ideas involved in Klyachko's proof. For any $\xi \in \mathfrak{t}_{+}$, let

$$
\mathcal{O}_{\xi}=S U(n) \cdot \xi=\{A \in \mathfrak{s u}(n) \mid \lambda(A)=\xi\}
$$

denote the corresponding adjoint orbit. Via the identification $\mathfrak{s u}(n) \cong \mathfrak{s u}(n)^{*}$, $\mathcal{O}_{\xi}$ inherits a canonical symplectic structure, called the Kostant-Kirillov-Souriau two-form, and the action of $S U(n)$ on $\mathcal{O}_{\xi}$ is Hamiltonian with moment map given by inclusion into $\mathfrak{s u}(n)$.

The diagonal action of $S U(n)$ on $\mathcal{O}_{\xi_{1}} \times \ldots \times \mathcal{O}_{\xi_{l}}$ has moment map given by

$$
\left(A_{1}, \ldots, A_{l}\right) \mapsto A_{1}+A_{2}+\ldots+A_{l} .
$$


The symplectic quotient $\mathcal{N}\left(\xi_{1}, \ldots, \xi_{l}\right)=\mathcal{O}_{\xi_{1}} \times \ldots \times \mathcal{O}_{\xi_{l}} / / S U(n)$ is given by

$$
\mathcal{N}\left(\xi_{1}, \ldots, \xi_{l}\right)=\left\{\left(A_{1}, \ldots, A_{l}\right) \in \mathcal{O}_{\xi_{1}} \ldots \mathcal{O}_{\xi_{l}} \mid A_{1}+A_{2}+\ldots+A_{l}=0\right\} / S U(n) .
$$

For generic $\left(\xi_{1}, \ldots, \xi_{l}\right)$, that is, values where the moment map has maximal rank, the quotient $\mathcal{N}\left(\xi_{1}, \ldots, \xi_{l}\right)$ is a symplectic manifold. The $l$-tuple $\left(\xi_{1}, \ldots, \xi_{l}\right)$ lies in $\Delta(l)$ if and only if $\mathcal{N}\left(\xi_{1}, \ldots, \xi_{l}\right)$ is non-empty.

The quotients $\mathcal{N}\left(\xi_{1}, \ldots, \xi_{l}\right)$ may be viewed as symplectic quotients of the cotangent bundle $T^{*} S U(n)^{l-1}$. Indeed, the symplectic quotient

$$
\left(T^{*} S U(n) \times \mathcal{O}_{\xi}\right) / / S U(n) \cong \mathcal{O}_{\xi}
$$

Therefore, the quotient of $T^{*} S U(n)^{l-1}$ by the right action of $S U(n)^{l-1}$ and the diagonal left action of $S U(n)$ is

$$
\left(T^{*} S U(n)^{l-1} \times \mathcal{O}_{\xi_{1}} \times \ldots \times \mathcal{O}_{\xi_{l}}\right) / / S U(n)^{l} \cong \mathcal{N}\left(\xi_{1}, \ldots, \xi_{l}\right) .
$$

It follows that $\Delta(l)$ is the moment polytope of the action of $S U(n)^{l}$ on $T^{*} S U(n)^{l-1}$.

One can determine whether $\mathcal{N}\left(\xi_{1}, \ldots, \xi_{l}\right)$ is empty by computing its symplectic volume. This is given by a formula due to G. Heckman [13] (see also $[11]$ or $[22,(4)])$. Unfortunately the formula involves cancellations and it is not apparent what the support of the volume function is, or even that the support is a convex polytope.

The manifolds $\mathcal{O}_{\xi_{i}}$ have canonical complex structures (induced by the choice of positive Weyl chamber) and are isomorphic to (possibly partial) flag varieties. Suppose that $\xi_{1}, \ldots, \xi_{l}$ lie in the weight lattice $\Lambda^{*}$, so that there exist prequantum line bundles $L_{\xi_{i}} \rightarrow \mathcal{O}_{\xi_{i}}$; i.e., equivariant line bundles with curvature equal to $2 \pi i$ times the symplectic form. The sections of $L_{\xi_{1}} \otimes \ldots \otimes L_{\xi_{l}}$ define a Kähler embedding

$$
\mathcal{O}_{\xi_{1}} \times \ldots \times \mathcal{O}_{\xi_{l}} \rightarrow \mathbb{P}\left(V_{\xi_{1}}^{*}\right) \times \ldots \times \mathbb{P}\left(V_{\xi_{l}}^{*}\right)
$$

where $V_{\xi_{1}}, \ldots, V_{\xi_{l}}$ are the irreducible representations whose highest weights are $\xi_{1}, \ldots, \xi_{l}$. By an application of a theorem of Kirwan and Kempf-Ness (which holds for arbitrary smooth projective varieties, see [17, page 109] or [9, Chapter $6]$ ) the symplectic quotient is homeomorphic to the geometric invariant theory quotient

$$
\mathcal{N}\left(\xi_{1}, \ldots, \xi_{l}\right) \cong \mathcal{O}_{\xi_{1}} \times \ldots \times \mathcal{O}_{\xi_{l}} / / S L(n, \mathbb{C}) .
$$

By definition, $\mathcal{O}_{\xi_{1}} \times \ldots \times \mathcal{O}_{\xi_{l}} / / S L(n, \mathbb{C})$ is the quotient of the set of semi-stable points in $\mathcal{O}_{\xi_{1}} \times \ldots \times \mathcal{O}_{\xi_{l}}$ by the action of $S L(n, \mathbb{C})$, where $\left(F_{\xi_{1}}, \ldots, F_{\xi_{l}}\right) \in$ $\mathcal{O}_{\xi_{1}} \times \ldots \times \mathcal{O}_{\xi_{l}}$ is called semi-stable if and only if for some $N$ there is an invariant section of $\left(L_{\xi_{1}} \otimes \ldots \otimes L_{\xi_{l}}\right) \otimes N$ which is non-vanishing at $\left(F_{\xi_{1}}, \ldots, F_{\xi_{l}}\right)$. The quotient $\mathcal{N}\left(\xi_{1}, \ldots, \xi_{l}\right)$ is therefore non-empty if and only if there exists a nonzero $S U(n)$-invariant vector in

$$
H^{0}\left(\left(L_{\xi_{1}} \otimes \ldots \otimes L_{\xi_{l}}\right)^{\otimes N}\right)=V_{N \xi_{1}} \otimes \ldots \otimes V_{N \xi_{l}} .
$$


This explains the representation-theoretic interpretation of $\Delta(l)$ alluded to in the introduction. ${ }^{2}$

In order to obtain the inequalities in Theorem 2.1, one applies the criterion of Mumford, which says that a point is semi-stable if and only if it is semi-stable for all one-parameter subgroups [25, Chapter 2]. (See also [17, Lemma 8.8] and $\left[9\right.$, Chapter 6].) Let us assume that the $\xi_{i}$ are generic. An application of the criterion gives that an $l$-tuple of complete flags $\left(F_{1}, \ldots, F_{l}\right) \in \mathcal{O}_{\xi_{1}} \times \ldots \times \mathcal{O}_{\xi_{l}}$ is semi-stable if and only if for all subspaces $W \subset \mathbb{C}^{n}$, one has

$$
\sum_{i \in I_{1}} \xi_{1, i}+\ldots+\sum_{i \in I_{l}} \xi_{l, i} \leq 0
$$

where $I_{j}$ is the position of $W$ with respect to the flag $F_{j}$. The proof similar to that for Grassmannians given in Section 4.4 of [25].

The set of semi-stable points is dense if it is non-empty. It follows that $\mathcal{N}\left(\xi_{1}, \ldots, \xi_{l}\right)$ is non-empty if and only if the above inequality holds for every intersection $\sigma_{I_{1}} \cap \ldots \cap \sigma_{I_{l}}$ of Schubert cycles in general position. Any inequality corresponding to a positive dimensional intersection must be redundant. Indeed, since the intersection is a projective variety, it cannot be contained in any of the Schubert cells. The boundary of $\sigma_{I_{l}}$ consists of Schubert varieties $\sigma_{J}$ with $J$ such that $j_{k} \leq i_{k}$ for $k=1, \ldots, r$, where $i_{1}, \ldots, i_{r}$ and $j_{1}, \ldots, j_{r}$ are the elements of $I_{l}$ and $J$ in increasing order. The inequality obtained from an intersection $\sigma_{I_{1}} \cap \ldots \cap \sigma_{I_{l-1}} \cap \sigma_{J} \neq \emptyset$ therefore implies the inequality that is obtained from $\sigma_{I_{1}} \cap \ldots \cap \sigma_{I_{l}} \neq \emptyset$.

\section{Application of the Mehta-Seshadri theorem}

For any $\xi \in \mathfrak{A}$, let

$$
\mathcal{C}_{\xi}=\{A \in S U(n) \mid \lambda(A)=\xi\}
$$

denote the corresponding conjugacy class. The mapping $A \mapsto \lambda(A)$ induces a homeomorphism $S U(n) / \operatorname{Ad}(S U(n)) \cong \mathfrak{A}$.

Let $p_{1}, \ldots, p_{l} \in \mathbb{P}^{1}$ be distinct marked points and $\mathcal{M}\left(\xi_{1}, \ldots, \xi_{l}\right)$ the moduli space of flat $S U(n)$-connections on $\mathbb{P}^{1} \backslash\left\{p_{1}, \ldots, p_{l}\right\}$ with holonomy around $p_{i}$ lying in $\mathcal{C}_{\xi_{i}}$. Since the fundamental group of $\mathbb{P}^{1} \backslash\left\{p_{1}, \ldots, p_{l}\right\}$ has generators the loops $\gamma_{1}, \ldots, \gamma_{l}$ around the punctures, with the single relation $\gamma_{1} \cdot \ldots \cdot \gamma_{l}=1$,

$$
\mathcal{M}\left(\xi_{1}, \ldots, \xi_{l}\right) \cong\left\{\left(A_{1}, \ldots, A_{l}\right) \in \mathcal{C}_{\xi_{1}} \times \ldots \times \mathcal{C}_{\xi_{l}} \mid A_{1} A_{2} \cdots A_{l}=I\right\} / S U(n) .
$$

In particular $\mathcal{M}\left(\xi_{1}, \ldots, \xi_{l}\right)$ is non-empty if and only if $\left(\xi_{1}, \ldots, \xi_{l}\right) \in \Delta_{q}(l)$. In theory one can determine if $\mathcal{M}\left(\xi_{1}, \ldots, \xi_{l}\right)$ is non-empty by computing its symplectic volume by the formulae stated in Witten [33, (4.11)], Szenes [31], and [23, Theorem 5.2].

\footnotetext{
${ }^{2}$ Recent work of Knutson and Tau [19] shows that one may take $N=1$ in this equation. Klyachko's work therefore yields a recursive algorithm for determining which irreducibles appear in a tensor product. This algorithm was originally conjectured by Horn.
} 
For rational $\xi_{1}, \ldots, \xi_{l}$ the space $\mathcal{M}\left(\xi_{1}, \ldots, \xi_{l}\right)$ has an algebro-geometric description due to Mehta-Seshadri [21]. Let $C$ be a Riemann surface with marked points $p_{1}, \ldots, p_{l} \in C$ and let $\mathcal{E} \rightarrow C$ be a holomorphic bundle. A parabolic structure without multiplicity on $\mathcal{E}$ consists of the following data at each marked point $p_{i}$ : a complete ascending flag

$$
0=\mathcal{E}_{p_{i}, 0} \subset \mathcal{E}_{p_{i}, 1} \subset \mathcal{E}_{p_{i}, 2} \ldots \subset \mathcal{E}_{p_{i}, n}=\mathcal{E}_{p_{i}},
$$

in the fiber $\mathcal{E}_{p_{i}}$, and a set of parabolic weights

$$
\lambda_{i, 1}>\lambda_{i, 2}>\ldots>\lambda_{i, n},
$$

satisfying $\lambda_{i, 1}-\lambda_{i, n} \leq 1$. In [21] the weights are required to lie in the interval $[0,1)$, but the definitions work without this assumption. A parabolic bundle is a holomorphic bundle with a parabolic structure. Recall that the degree $\operatorname{deg}(\mathcal{E})$ of $\mathcal{E}$ is the first Chern class $c_{1}(\mathcal{E}) \in H^{2}(C, \mathbb{Z}) \cong \mathbb{Z}$. The parabolic degree pardeg $(\mathcal{E})$ is defined by

$$
\operatorname{pardeg}(\mathcal{E})=\operatorname{deg}(\mathcal{E})+\sum_{i=1, j=1}^{l, n} \lambda_{i, j} .
$$

The parabolic slope $\mu(\mathcal{E})$ is

$$
\mu(\mathcal{E})=\frac{\operatorname{pardeg}(\mathcal{E})}{\operatorname{rk}(\mathcal{E})} .
$$

Given a holomorphic sub-bundle $\mathcal{F} \subset \mathcal{E}$ of rank $r$ one obtains a parabolic structure on $\mathcal{F}$ as follows. An ascending flag in the fiber $\mathcal{F}_{p_{i}}$ at each marked point $p_{i}$ is obtained by removing from

$$
\mathcal{F}_{p_{i}} \cap \mathcal{E}_{p_{i}, 1} \subseteq \mathcal{F}_{p_{i}} \cap \mathcal{E}_{p_{i}, 2} \subseteq \ldots \subseteq \mathcal{F}_{p_{i}} \cap \mathcal{E}_{p_{i}, n}=\mathcal{F}_{p_{i}},
$$

those terms for which the inclusion is not strict. The parabolic weights for $\mathcal{F}$ are

$$
\mu_{i, j}=\lambda_{i, k_{j}}
$$

where $k_{j}$ is the minimal index such that $\mathcal{F}_{p_{i}, j} \subseteq \mathcal{E}_{p_{i}, k_{j}}$. Let $K_{i}=\left\{k_{1}, \ldots, k_{r}\right\}$. The fiber $\mathcal{F}_{p_{i}}$ may be viewed as a element of the Grassmannian of $r$-planes in $\mathcal{E}_{p_{i}}$, and $K$ is the position of $\mathcal{F}_{p_{i}}$ with respect to the flag $\mathcal{E}_{p_{i}, *}$. The parabolic degree of $\mathcal{F}$ is

$$
\operatorname{pardeg}(\mathcal{F})=\operatorname{deg}(\mathcal{F})+\sum_{i, k \in K_{i}} \lambda_{i, k}
$$

A parabolic sub-bundle of $\mathcal{E}$ is a holomorphic sub-bundle $\mathcal{F} \subset E$ whose parabolic structure is the one induced from the inclusion. A parabolic bundle $\mathcal{E} \rightarrow C$ is called parabolic semi-stable if $\mu(\mathcal{F}) \leq \mu(\mathcal{E})$ for all parabolic subbundles $\mathcal{F} \subset \mathcal{E}$. There is a natural equivalence relation on parabolic bundles: Two bundles are said to be grade equivalent if the associated graded bundles are isomorphic as parabolic bundles. See [21] for more details. 
Theorem 5.1 (Mehta-Seshadri). Suppose the parabolic weights $\lambda_{i, j}$ are $r a-$ tional and lie in the interval $[0,1)$. Then the moduli space $\mathcal{M}\left(\lambda_{1}, \ldots, \lambda_{l}\right)$ of grade equivalence classes of semi-stable parabolic bundles with parabolic weights $\lambda_{i, j}$ and parabolic degree 0 is a normal, projective variety, homeomorphic to the moduli space of flat unitary connections over $C \backslash\left\{p_{1}, \ldots, p_{r}\right\}$ such that the holonomy of a small loop around $p_{i}$ lies in $\mathcal{C}_{\lambda_{i}}$.

In fact, the Mehta-Seshadri theorem also holds without the assumption that the parabolic weights lie in $[0,1)$. One can see this either through the theory of elementary transformations, or through the extension of the Mehta-Seshadri theorem given in Boden [7].

The explanation using elementary transformations goes as follows. Let $\mathcal{Q}$ denote the skyscraper sheaf with fiber $\mathcal{E}_{p_{i}} / \mathcal{E}_{p_{i}, n-1}$ at $p_{i}$. One has an exact sequence of sheaves

$$
0 \rightarrow \mathcal{E}^{\prime} \rightarrow \mathcal{E} \rightarrow \mathcal{Q} \rightarrow 0
$$

The kernel $\mathcal{E}^{\prime}$ is a sub-sheaf of a locally free sheaf and therefore locally free. Since degree is additive in short exact sequences $\operatorname{deg}\left(\mathcal{E}^{\prime}\right)=\operatorname{deg}(\mathcal{E})-1$. One calls the $\mathcal{E}^{\prime}$ an elementary transformation of $\mathcal{E}$ at $p_{i}$. There is a canonical line $\mathcal{E}_{p_{i, 1}}^{\prime}$ in the fiber $\mathcal{E}_{p_{i}}^{\prime}$ which is the kernel of the fiber map $\pi: \mathcal{E}_{p_{i}}^{\prime} \rightarrow \mathcal{E}_{p_{i}}$. One extends the canonical line to a complete flag by taking $\mathcal{E}_{p_{i}, j}^{\prime}=\pi^{-1}\left(\mathcal{E}_{p_{i}, j-1}^{\prime}\right)$ for $j>1$. Finally one takes as parabolic weights at $p_{i}$ the set $\lambda_{i, n}+1, \lambda_{i, 1}, \ldots, \lambda_{i, n-1}$. With this parabolic structure the bundle $\mathcal{E}^{\prime}$ is parabolic semi-stable of the same parabolic degree as $\mathcal{E}$. Details, in a slightly different form, can be found in Boden and Yokogawa [8].

The following is the key lemma in the derivation of Theorem 5.3 from MehtaSeshadri. Let $d=\operatorname{deg}(\mathcal{E})=-\sum \lambda_{i, j}$ denote the degree of any element $\mathcal{E} \in$ $\mathcal{M}\left(\lambda_{1}, \ldots, \lambda_{l}\right)$.

Lemma 5.2. Suppose that there is some ordinary semi-stable bundle on $C$ of degree $d$. Then the set of equivalence classes of parabolic semi-stable bundles of parabolic degree 0 whose underlying holomorphic bundle is ordinary semi-stable is Zariski dense in $\mathcal{M}\left(\lambda_{1}, \ldots, \lambda_{l}\right)$.

Proof. Recall from the construction of $\mathcal{M}\left(\lambda_{1}, \ldots, \lambda_{l}\right)$ in [21] that for some integer $N$ there exists an $S L(N)$-equivariant bundle $\tilde{R} \stackrel{\pi}{\rightarrow} R$ whose fibers are products of $l$ complete flag varieties, such that the geometric invariant theory quotients of $\tilde{R}, R$ are

$$
\tilde{R} / / S L(N)=\mathcal{M}\left(\lambda_{1}, \ldots, \lambda_{l}\right), \quad R / / S L(N)=\mathcal{M}
$$

where $\mathcal{M}$ denotes the moduli space of ordinary semi-stable bundles on $C$ of degree $d$. By definition,

$$
\tilde{R} / / S L(N)=\tilde{R}^{\mathrm{ss}} / S L(N), \quad R / / S L(N)=R^{\mathrm{ss}} / S L(N)
$$


where $\tilde{R}^{\mathrm{ss}}, R^{\mathrm{ss}}$ denote the Zariski dense set of semi-stable points in $\tilde{R}, R$ respectively. The inverse image $\pi^{-1}\left(R^{\mathrm{ss}}\right) \cap \tilde{R}^{\mathrm{ss}} / S L(N)$ is thus dense in $\tilde{R}^{\mathrm{ss}} / S L(N)=$ $\mathcal{M}\left(\lambda_{1}, \ldots, \lambda_{l}\right)$.

Now we specialize to the case $C=\mathbb{P}^{1}$ with $l$ marked points $p_{1}, p_{2}, \ldots, p_{l}$. Let $\xi_{1}, \ldots, \xi_{l} \in \mathfrak{A}^{l} \cap \mathbb{Q}^{l}$. By Lemma $5.2, \mathcal{M}\left(\xi_{1}, \ldots, \xi_{l}\right)$ is non-empty if and only there exists a parabolic semi-stable $\mathcal{E}$ with parabolic degree 0 and weights $\xi_{1}, \ldots, \xi_{l}$ whose underlying holomorphic bundle is semi-stable. Since the sum of the parabolic weights is zero, the degree of $\mathcal{E}$ is also zero. By Grothendieck's theorem, $\mathcal{E}$ is holomorphically trivial. A sub-bundle $\mathcal{F} \subset \mathcal{E}$ of rank $r$ is given by a holomorphic map

$$
\varphi_{\mathcal{F}}: \mathbb{P}^{1} \rightarrow G(r, n)
$$

Since $\varphi_{\mathcal{F}}$ is the classifying map of the quotient $\mathcal{E} / \mathcal{F}$, the degree of $\mathcal{F}$ is minus the degree of $\varphi_{\mathcal{F}}$. The parabolic slope of $\mathcal{F}$ is given by

$$
\mu(\mathcal{F})=\frac{1}{r}\left(-\operatorname{deg}\left(\varphi_{\mathcal{F}}\right)+\sum_{i \in I_{1}(\varphi)} \xi_{1, i}+\ldots+\sum_{i \in I_{1}(\varphi)} \xi_{l, i}\right),
$$

where $I_{i}(\varphi)$ is the position of the subspace $\varphi\left(p_{i}\right) \subset \mathcal{E}_{p_{i}}$ with respect to the flag $\mathcal{E}_{p_{i}, *}$ above. The parabolic bundle $\mathcal{E}$ is called parabolic semi-stable if and only if for all such $F, \mu(F) \leq 0$, that is,

$$
\sum_{i \in I_{1}(\varphi)} \xi_{1, i}+\ldots+\sum_{i \in I_{1}(\varphi)} \xi_{l, i} \leq \operatorname{deg}(\varphi)
$$

for all maps $\varphi: \mathbb{P}^{1} \rightarrow G(r, n)$.

The following result was obtained independently by P. Belkale [2].

Theorem 5.3. A complete set of inequalities for $\Delta_{q}(l)$ as a subset of $\mathfrak{A}^{l}$ is given by

$$
\sum_{i \in I_{1}} \lambda_{i}\left(A_{1}\right)+\sum_{i \in I_{2}} \lambda_{i}\left(A_{2}\right)+\ldots+\sum_{i \in I_{l}} \lambda_{i}\left(A_{l}\right) \leq d
$$

for subsets $I_{1}, \ldots, I_{l} \subset\{1, \ldots, n\}$ of the same cardinality $r$ and non-negative integers $d$ such that there exists a rational map $\mathbb{P}^{1} \rightarrow G(r, n)$ of degree $d$ mapping $p_{1}, \ldots, p_{l}$ to the Schubert cells $C_{I_{1}}, \ldots, C_{I_{l}}$ in general position.

Proof. If $\mathcal{M}\left(\xi_{1}, \ldots, \xi_{l}\right)$ is non-empty, then a trivial bundle with a general choice of flags will be parabolic semi-stable. Indeed, by the above discussion the fiber Flag $^{l}$ of $\pi: \tilde{R} \rightarrow R$ over a trivial bundle intersects $\tilde{R}^{s s}$, so $\tilde{R}^{s s} \cap \mathrm{Flag}^{l}$ is open in Flag ${ }^{l}$. Therefore, $\mathcal{M}\left(\xi_{1}, \ldots, \xi_{l}\right)$ is non-empty if and only if

$$
\sum_{i \in I_{1}} \xi_{1, i}+\ldots+\sum_{i \in I_{l}} \xi_{l, i} \leq d
$$

for all subsets $I_{1}, \ldots, I_{l}$ and integers $d$ such that there exists a degree $d$ map sending $p_{1}, \ldots, p_{l}$ to general translates of the Schubert cells $C_{I_{1}}, \ldots, C_{I_{l}}$. 
Remark 5.4. For sufficiently small parabolic weights $\lambda_{i, j}$ any parabolic semistable bundle on $\mathbb{P}^{1}$ is necessarily ordinary semi-stable of degree 0 , and therefore trivial. It follows that the moduli spaces $\mathcal{M}\left(\lambda_{1}, \ldots, \lambda_{l}\right)$ and $\mathcal{N}\left(\lambda_{1}, \ldots, \lambda_{l}\right)$ are isomorphic. This shows that Klyachko's result is implied by Theorem 5.3.

We now show that the existence of the maps described in Theorem 5.3 may be detected by Gromov-Witten invariants. Let $\sigma_{I_{1}}, \ldots, \sigma_{I_{l}}$ be some collection of Schubert varieties, and consider the expansion

$$
\left[\sigma_{I_{1}}\right] \star\left[\sigma_{I_{2}}\right] \ldots \star\left[\sigma_{I_{l}}\right]=\sum_{i} q^{i} \alpha_{i}
$$

where $\alpha_{i} \in H_{*}(G(r, n)){ }^{3}$ We say that $q^{d}$ divides $\left[\sigma_{I_{1}}\right] \star\left[\sigma_{I_{2}}\right] \ldots \star\left[\sigma_{I_{l}}\right]$ if $\alpha_{i}=0$ for all $i<d$. The following lemma is stated in Ravi [28].

Lemma 5.5. Let $d$ be the lowest degree of a map $\mathbb{P}^{1} \rightarrow G(r, n)$ sending $p_{1}, \ldots, p_{l}$ to general translates of $\sigma_{I_{1}}, \ldots, \sigma_{I_{l}}$ respectively. Then $q^{d}$ is the maximal power of $q$ dividing $\left[\sigma_{I_{1}}\right] \star \ldots \star\left[\sigma_{I_{l}}\right]$.

Proof. Let $\mathcal{M}_{d}$ denote the space of maps $\mathbb{P}^{1} \rightarrow G(r, n)$ of degree $d$, ev ${ }^{l}: \mathcal{M}_{d} \rightarrow$ $G(r, n)^{l}$ the evaluation map, and $\sigma_{I_{*}}\left(p_{*}\right)=\left(\mathrm{ev}^{l}\right)^{-1}\left(\sigma_{I_{*}}\right)$ the subset of maps sending $p_{j}$ to $\sigma_{I_{j}}$ for $j=1, \ldots, l$. By [3, Moving Lemma 2.2A], $\sigma_{I_{*}}\left(p_{*}\right)$ is a quasi-projective variety, of the expected codimension in $\mathcal{M}_{d}$. By choosing enough additional marked points $p_{1}^{\prime}, \ldots, p_{m}^{\prime}$, we can insure that the corresponding evaluation map ev ${ }^{m}: \mathcal{M}_{d} \rightarrow G(r, n)^{m}$ is injective when restricted to $\sigma_{I_{*}}\left(p_{*}\right)$. Let $Y \subset G(r, n)^{l} \times G(r, n)^{m}$ be the closure of $\left(\mathrm{ev}^{l} \times \mathrm{ev}^{m}\right)\left(\mathcal{M}_{d}\right)$, and let $\phi: G(r, n)^{l} \times G(r, n)^{m} \rightarrow G(r, n)^{m}$ be the projection.

Since the homology class $\left[\phi\left(Y \cap \sigma_{I_{*}}\right)\right]$ is non-trivial [10, page 64], $\phi\left(Y \cap \sigma_{I_{*}}\right)$ must intersect some Schubert variety

$$
\sigma_{J_{*}}=\sigma_{J_{1}} \times \sigma_{J_{2}} \times \ldots \times \sigma_{J_{m}} \subset G(r, n)^{m}
$$

of complementary dimension. By Kleiman's lemma, [12, Theorem 10.8 page 273], the singular locus of $\phi\left(Y \cap \sigma_{I_{*}}\right)$ does not intersect a general translate of $\sigma_{J_{*}}$, and similarly the singular locus of $\sigma_{J_{*}}$ does not intersect $\phi\left(Y \cap \sigma_{I_{*}}\right)$. Therefore the intersection occurs in the smooth loci of $\phi\left(Y \cap \sigma_{I_{*}}\right)$ and $\sigma_{J_{*}}$, and another application of the lemma implies that the intersection is finite.

For generic translates of $\sigma_{J_{*}}$, the intersection is contained in $\mathrm{ev}^{m}\left(\sigma_{I_{*}}\left(p_{*}\right)\right)$. Indeed, let $\bar{\sigma}_{I_{*}}\left(p_{*}\right)$ be the compactification of $\sigma_{I_{*}}\left(p_{*}\right)$ given in [3], and $\Gamma \subset$ $\bar{\sigma}_{I_{*}}\left(p_{*}\right) \times G(r, n)^{m}$ the closure of the graph of ev ${ }^{m}$. Let $Z \subset \Gamma$ be the complement of the graph of ev ${ }^{m}$. The projection $\pi(Z)$ of $Z$ in $G(r, n)^{m}$ is a closed sub-variety of $\phi\left(Y \cap \sigma_{I_{*}}\right)$. By Kleiman's lemma, for generic translates of $\sigma_{J_{*}}$ the intersection of $\pi(Z)$ and $\sigma_{J_{*}}$ is empty, so the intersection is contained in $\mathrm{ev}^{m}\left(\sigma_{I_{*}}\left(p_{*}\right)\right)$.

\footnotetext{
${ }^{3}$ The following argument shows that this product is always non-zero. Multiply the left-hand side by the dual classes $\left[\sigma_{* I_{1}}\right], \ldots,\left[\sigma_{* I_{l}}\right]$. The leading term in this quantum product is the $l$-th quantum power of the class of a point, $[p t]^{\star l}$. It is easy to verify, using the techniques of Section 7 , that this product is non-zero. Therefore, $\left[\sigma_{I_{1}}\right] \star \ldots \star\left[\sigma_{I_{l}}\right]$ is also non-zero.
} 
Because ev ${ }^{m} \mid \sigma_{I_{*}}\left(p_{*}\right)$ is injective, the intersection $\sigma_{I_{*}}\left(p_{*}\right) \cap \sigma_{J_{*}}\left(p_{*}^{\prime}\right)$ is finite and non-empty. Since the homology class $\left[\phi\left(Y \cap \sigma_{I_{*}}\right)\right]$ is independent of the choice of general translate of $\sigma_{I_{*}}$, the above intersection is finite and non-empty for general translates of the $\sigma_{I_{i}}$ and $\sigma_{J_{j}}$. This implies that Gromov-Witten invariant

$$
\left\langle\left[\sigma_{I_{1}}\right], \ldots,\left[\sigma_{I_{l}}\right],\left[\sigma_{J_{1}}\right], \ldots,\left[\sigma_{J_{m}}\right]\right\rangle_{d} \neq 0
$$

In terms of the quantum product

$$
\left[\sigma_{I_{1}}\right] \star \ldots \star\left[\sigma_{I_{l}}\right] \star\left[\sigma_{J_{1}}\right] \star \ldots \star\left[\sigma_{J_{m-1}}\right] \supset q^{d} \sigma_{* J_{m}},
$$

which implies that $\left[\sigma_{I_{1}}\right] \star \ldots \star\left[\sigma_{I_{l}}\right]$ contains a term with coefficient $q^{i}$ with $i \leq d$. That is, for some Schubert variety $\sigma$,

$$
\left\langle\left[\sigma_{I_{1}}\right],\left[\sigma_{I_{2}}\right], \ldots,\left[\sigma_{I_{l}}\right],[\sigma]\right\rangle_{i} \neq 0 .
$$

To prove the lemma it suffices to show that $i=d$. By [3, Moving Lemma 2.2 ], for general translates of the Schubert varieties the degree $i$ moduli space $\sigma_{I_{1}}\left(p_{1}\right) \cap \ldots \cap \sigma_{I_{l}}\left(p_{l}\right) \cap \sigma(p)$ is finite and consists of maps sending $p_{1}, \ldots, p_{l}, p$ to the corresponding Schubert cells. Since $d$ is minimal, $i=d$.

\section{Factorization}

In this section we show that a relationship between the polytopes for different numbers of marked points is related to factorization of Gromov-Witten invariants (i.e. associativity of quantum multiplication). A similar, easier, discussion holds for the additive polytopes $\Delta(l)$. A consideration of a "trivial" factorization completes the proof of Theorem 3.1.

Suppose that $l$ can be written $l=j+k-2$ for positive integers $j, k \geq 2$. It is easy to see that $\Delta_{q}(l)$ are projections of a section of $\Delta_{q}(j) \times \Delta_{q}(k)^{4}$.

$$
\Delta_{q}(l)=\left\{\left(\mu_{1}, \ldots, \mu_{j-1}, \nu_{1}, \ldots, \nu_{k-1}\right) \mid(\mu, \nu) \in \Delta_{q}(j) \times \Delta_{q}(k), \quad \mu_{j}=* \nu_{k}\right\}
$$

To show the forward inclusion, note that if $A_{1} A_{2} \ldots A_{l}=I$ then letting $B=$ $A_{j} A_{j+1} \ldots A_{l}$ we have

$$
\begin{aligned}
& \left(\lambda\left(A_{1}\right), \ldots, \lambda\left(A_{j-1}\right), \lambda(B)\right) \in \Delta_{q}(j), \\
& \left(\lambda\left(B^{-1}\right), \lambda\left(A_{j}\right), \ldots, \lambda\left(A_{l}\right)\right) \in \Delta_{q}(k) .
\end{aligned}
$$

\footnotetext{
${ }^{4}$ In fact, the volume functions satisfy the factorization properties

$\operatorname{Vol}\left(\mathcal{N}\left(\mu_{1}, \ldots, \mu_{j-1}, \nu_{1}, \ldots, \nu_{k-1}\right)\right)=$$$
\int_{\mathrm{t}+} \operatorname{Vol}\left(\mathcal{N}\left(\mu_{1}, \ldots, \mu_{j-1}, * \lambda\right)\right) \operatorname{Vol}\left(\mathcal{N}\left(* \lambda, \nu_{1}, \ldots, \nu_{k-1}\right)\right) \mathrm{d} \lambda,
$$

$\operatorname{Vol}\left(\mathcal{M}\left(\mu_{1}, \ldots, \mu_{j-1}, \nu_{1}, \ldots, \nu_{k-1}\right)\right)=$

$$
\int_{\mathfrak{l}} \operatorname{Vol}\left(\mathcal{M}\left(\mu_{1}, \ldots, \mu_{j-1}, * \lambda\right)\right) \operatorname{Vol}\left(\mathcal{M}\left(* \lambda, \nu_{1}, \ldots, \nu_{k-1}\right)\right) \mathrm{d} \lambda .
$$
}

The second formula is implicit in Witten [33, p.51], proved in [16], and generalized in [24]. 
In particular this means that any face of $\Delta_{q}(l)$ is a projection of a face (usually not of codimension 1 ) of $\Delta_{q}(j) \times \Delta_{q}(k)$. Any face is the intersection of codimension 1 faces. This shows that any defining inequality of $\Delta_{q}(l)$ is implied by a finite set of defining inequalities for $\Delta_{q}(j)$ and $\Delta_{q}(k)$.

Using associativity of quantum cohomology one can be more specific about which inequalities for $\Delta_{q}(j), \Delta_{q}(k)$ are needed to imply an inequality for $\Delta_{q}(l)$. Suppose that a Gromov-Witten invariant $\left\langle\sigma_{I_{1}}, \ldots, \sigma_{I_{l}}, \sigma_{J}\right\rangle_{d} \neq 0$ so that one has an inequality for $\Delta(l)$ given by

$$
\sum_{i \in I_{1}} \lambda_{1, i}+\ldots+\sum_{i \in I_{l}} \lambda_{l, i} \leq d
$$

Associativity of quantum multiplication says that

$$
\begin{aligned}
& \left\langle\sigma_{I_{1}}, \ldots, \sigma_{I_{l}}, \sigma_{J}\right\rangle_{d}= \\
& \sum_{d_{1}+d_{2}=d,|K|=r}\left\langle\sigma_{I_{1}}, \ldots, \sigma_{I_{j-1}}, \sigma_{K}\right\rangle_{d_{1}}\left\langle\sigma_{* K}, \sigma_{I_{j}}, \ldots, \sigma_{I_{l}}, \sigma_{J}\right\rangle_{d_{2}} .
\end{aligned}
$$

In particular there exist some $d_{1}, d_{2}$ with $d_{1}+d_{2}=d$ and some Schubert variety $\sigma_{K}$ such that

$$
\left\langle\sigma_{I_{1}}, \ldots, \sigma_{I_{j-1}}, \sigma_{K}\right\rangle_{d_{1}} \neq 0, \quad\left\langle\sigma_{* K}, \sigma_{I_{j}}, \ldots, \sigma_{I_{l}}, \sigma_{J}\right\rangle_{d_{2}} \neq 0 .
$$

From the non-vanishing of these Gromov-Witten invariants one deduces the inequalities for $\Delta_{q}(j), \Delta_{q}(k)$ :

$$
\begin{gathered}
\sum_{i \in I_{1}} \mu_{1, i}+\ldots+\sum_{i \in I_{j-1}} \mu_{j-1, i}+\sum_{k \in K} \mu_{j, k} \leq d_{1} ; \\
\sum_{k \in * K} \nu_{1, k}+\sum_{i \in I_{j}} \nu_{2, i}+\ldots+\sum_{i \in I_{l}} \nu_{k, i} \leq d_{2} .
\end{gathered}
$$

Restricting to the section $\mu_{j}=* \nu_{1}$ one has that

$$
\sum_{k \in * K} \nu_{1, k}=-\sum_{k \in K}\left(* \nu_{1}\right)_{k}=-\sum_{k \in K} \mu_{j, k}
$$

so by adding the two inequalities one obtains (13).

Using the trivial factorization $l=(l+2)-2$ we complete the proof of Theorem 3.1 .

Lemma 6.1. Any inequality for $\Delta_{q}(l)$ corresponding to a Gromov-Witten invariant

$$
\left\langle\left[\sigma_{I_{1}}\right], \ldots,\left[\sigma_{I_{l}}\right],\left[\sigma_{K}\right]\right\rangle_{d} \neq 0,
$$

is a consequence of an inequality corresponding to a Gromov-Witten invariant of the form

$$
\left\langle\left[\sigma_{I_{1}}\right], \ldots,\left[\sigma_{I_{l-1}}\right],\left[\sigma_{J}\right]\right\rangle_{d_{1}} \neq 0,
$$

for some $J \subset\{1, \ldots, n\}$ and $d_{1} \leq d$. 
Proof. Suppose that

$$
\left\langle\left[\sigma_{I_{1}}\right], \ldots,\left[\sigma_{I_{l}}\right],\left[\sigma_{K}\right]\right\rangle_{d} \neq 0
$$

Taking $k=2$ we obtain that for some $J$ and $d_{1} \leq d$

$$
\left\langle\left[\sigma_{I_{1}}\right], \ldots,\left[\sigma_{I_{l-1}}\right],\left[\sigma_{J}\right]\right\rangle_{d_{1}}\left\langle\left[\sigma_{* J}\right],\left[\sigma_{I_{l}}\right],\left[\sigma_{K}\right]\right\rangle_{d_{2}} \neq 0 .
$$

Thus the inequality

$$
\sum_{i \in I_{1}} \lambda_{1, i}+\ldots+\sum_{i \in I_{l}} \lambda_{l, i} \leq d
$$

follows from the inequalities

$$
\sum_{i \in I_{1}} \lambda_{1, i}+\ldots+\sum_{i \in I_{l-1}} \lambda_{l-1, i}+\sum_{j \in J} \lambda_{l, j} \leq d_{1}
$$

for $\lambda \in \Delta_{q}(l)$, and

$$
\sum_{j \in * J}\left(* \lambda_{l}\right)_{j}+\sum_{i \in I_{l}}\left(\lambda_{l}\right)_{i} \leq d_{2}
$$

The last equation is a tautology for $\lambda_{l} \in \mathfrak{A}$ by the $l=2$ case of Theorem 5.3. In other words, (19) is implied by the equations $\lambda_{l, i} \geq \lambda_{l, i+1}, \lambda_{l, 1}-\lambda_{l, n} \leq 1$. Thus (17) follows from (18) and the inequalities defining $\mathfrak{A}^{l}$.

\section{Hidden symmetry}

An interesting aspect of the multiplicative problem is that it possesses a symmetry not present in the additive case, related to the symmetry of the fundamental alcove $\mathfrak{A}$ of $S U(n)$. Let $Z \cong \mathbb{Z} / n \mathbb{Z}$ denote the center of $S U(n)$, with generator $c \in S U(n)$ the unique element of $S U(n)$ with

$$
\lambda(c)=(1 / n, 1 / n, \ldots, 1 / n,(1-n) / n) .
$$

The action of $Z$ on $S U(n)$ induces an action on $\mathfrak{A} \cong S U(n) / \operatorname{Ad}(S U(n))$, given by

$$
c \cdot\left(\lambda_{1}, \ldots, \lambda_{n}\right)=\left(\lambda_{2}+1 / n, \lambda_{3}+1 / n, \ldots, \lambda_{n}+1 / n, \lambda_{1}-(n-1) / n\right) .
$$

Let $C(l) \subset S U(n)^{l}$ denote the subgroup

$$
C(l)=\left\{\left(z_{1}, \ldots, z_{l}\right) \subset Z^{l} \mid z_{1} z_{2} \ldots z_{l}=1\right\} \cong Z^{l-1} .
$$

The action of $C(l)$ on $\mathfrak{A}^{l}$ leaves the polytope $\Delta_{q}(l)$ invariant.

This symmetry of the polytope $\Delta_{q}(l)$ implies a symmetry on the facets of $\Delta_{q}(l)$. Let $c$ act on subsets of $\{1,2, \ldots, n\}$ via the action of $(12 \ldots n)^{-1} \in S_{n}$ :

$$
c^{m}\left\{i_{1}, \ldots, i_{r}\right\}=\left\{i_{s+1}-m, \ldots, i_{r}-m, i_{1}-m+n, \ldots, i_{s}-m+n\right\},
$$

where $s$ is the largest index for which $i_{s}-m \geq 1$ 
Suppose an $l+1$-tuple $\left(I_{1}, \ldots, I_{l}, d\right)$ defines a facet of $\Delta_{q}(l)$ via the inequality (10). Under the action of $\left(c^{m_{1}}, \ldots, c^{m_{l}}\right) \in C(l)$, (10) becomes the inequality corresponding to $\left(c^{m_{1}} I_{1}, \ldots, c^{m_{l}} I_{l}, d^{\prime}\right)$ where $d^{\prime}$ is defined by

$$
\sum_{i=1}^{l}\left|c^{m_{i}} I_{i}\right|+n d^{\prime}=\sum_{i=1}^{l}\left|I_{i}\right|+n d .
$$

Example 7.1. From the degree 0 inequality $\lambda_{n}(A)+\lambda_{n}(B) \leq \lambda_{n}(A B)$ we obtain by the action of $\left(c^{-i}, c^{-j}, c^{i+j}\right), i+j \leq n$ the degree 1 inequality (8).

Equation (20) defines a $C(l)$ action on the set of $l+1$-tuples $\left(I_{1}, \ldots, I_{l}, d\right)$ defining facets of $\Delta_{q}(l)$. It is an interesting fact that the Gromov-Witten invariants $\left\langle\sigma_{I_{1}}, \ldots, \sigma_{I_{l}}\right\rangle_{d}$ are invariant under this action:

Proposition 7.2. Let $\left(c^{m_{1}}, \ldots, c^{m_{l}}\right) \in C(l)$. Then,

$$
\left\langle\sigma_{I_{1}}, \ldots, \sigma_{I_{l}}\right\rangle_{d}=\left\langle\sigma_{c^{m_{1} I_{1}}}, \ldots, \sigma_{c^{m_{l}} I_{l}}\right\rangle_{d^{\prime}} .
$$

Proof. Let $\sigma_{c}=\sigma_{r, r+1, \ldots, n-1}$ denote the Schubert variety isomorphic to the Grassmannian $G(r, n-1)$ of $r$-planes contained in $n-1$-space. We claim that quantum multiplication by $\sigma_{c}$ is given by the following formula:

$$
\left[\sigma_{c}\right] \star\left[\sigma_{I}\right]=q^{(|c I|+r-|I|) / n}\left[\sigma_{c I}\right] .
$$

The exponent $(|c I|+r-|I|) / n$ equals 1 if $1 \in I$, and equals 0 otherwise. In particular $\left[\sigma_{c}\right]^{\star n}=q^{r}$.

The lemma then follows by associativity of the quantum product. Without loss of generality it suffices to show that the Gromov-Witten invariants are invariant under an element of the form $\left(c, c^{-1}, 1, \ldots, 1\right) \in C(l)$. Given that

$$
\left[\sigma_{I_{1}}\right] \star \ldots \star\left[\sigma_{I_{l-1}}\right] \supset\left\langle\sigma_{I_{1}}, \ldots, \sigma_{I_{l}}\right\rangle_{d}\left[\sigma_{* I_{l}}\right] q^{d},
$$

multiplying by $\left[\sigma_{c}\right]$ on both sides yields

$$
\left[\sigma_{c I_{1}}\right] \star \ldots \star\left[\sigma_{I_{l-1}}\right] \supset\left\langle\sigma_{I_{1}}, \ldots, \sigma_{I_{l}}\right\rangle_{d}\left[\sigma_{c\left(* I_{l}\right)}\right] q^{d^{\prime}}=\left\langle\sigma_{I_{1}}, \ldots, \sigma_{I_{l}}\right\rangle_{d}\left[\sigma_{* c^{-1} I_{l}}\right] q^{d^{\prime}} .
$$

The formula (21) may be proved using either the canonical isomorphism of quantum Schubert calculus with the Verlinde algebra of $U(r)$,

$$
Q H^{*}(G(r, n)) /(q=1) \cong R\left(U(r)_{n-r, n}\right) .
$$

given a mathematical proof in Agnihotri [1], or using the combinatorial formula of Bertram, Ciocan-Fontanine and Fulton [4]. $R\left(U(r)_{n-r, n}\right)$ denotes the Verlinde algebra of $U(r)$ at $S U(r)$ level $n-r$ and $U(1)$ level $n$, and is the quotient of the tensor algebra $R(U(r))$ by the relations

$$
V_{\lambda} \sim(-1)^{l(w)} V_{w(\lambda+\rho)-\rho}, w \in W_{\mathrm{aff}},
$$

and if $\lambda_{1}-\lambda_{r} \leq n-r$, then

$$
V_{\left(\lambda_{1}, \ldots, \lambda_{r}\right)} \sim V_{\left(\lambda_{2}-1, \lambda_{3}-1, \ldots, \lambda_{r}-1, \lambda_{1}-(n-r+1)\right.} .
$$


Here $W_{\text {aff }}$ acts on $\Lambda^{*}$ at level $n$, and $\rho$ is the half-sum of positive roots. The Verlinde algebra $R\left(U(r)_{n-r, n}\right)$ has as a basis the (equivalence classes of the) representations $V_{\lambda}$, where $\lambda=\left(\lambda_{1}, \ldots, \lambda_{r}\right) \in \mathbb{Z}^{r}, 0 \leq \lambda_{i} \leq n-r$ are dominant weights of $U(r)$ at level $n-r$.

The canonical isomorphism is given by $\sigma_{I} \mapsto V_{\lambda}$, where $\lambda$ is defined by

$$
\lambda_{j}=n-r+j-i_{j}
$$

The key point is that the sub-algebra $R(U(1)) \subset R(U(r))$ descends to a sub-algebra $R\left(U(1)_{n}\right) \subset R\left(U(r)_{n-r, r}\right)$ generated by the representation $V_{c}:=$ $V_{(1,1, \ldots, 1)}$, which maps under the isomorphism to the Schubert variety $\sigma_{c}$. From the description of the algebra given above one sees that $V_{c} \circledast V_{\lambda}=V_{\lambda^{\prime}}$ where

$$
\lambda^{\prime}=\begin{array}{cc}
\left(\lambda_{1}+1, \lambda_{2}+1, \ldots, \lambda_{r}+1\right) & \text { if } \lambda_{1}<n-r \\
\left(\lambda_{2}, \ldots, \lambda_{r}, \lambda_{1}-n+r\right) & \text { if } \lambda_{1}=n-r
\end{array} .
$$

Since $V_{\lambda^{\prime}}$ maps to $\sigma_{c I}$ under the canonical isomorphism, this proves (21).

Alternatively, (21) can be derived from the combinatorial rim-hook formula of $\left[4\right.$, p. 8]. Let $\lambda^{t}$ denote the transpose of $\lambda$, so that $\sigma_{\lambda^{t}}$ is the image of $\sigma_{\lambda}$ under the isomorphism $G(r, n) \cong G(n-r, n)$. The ordinary (resp. quantum) Littlewood-Richardson numbers are invariant under transpose

$$
N_{\lambda^{t} \mu^{t}}^{\rho^{t}}=N_{\lambda \mu}^{\rho}, \quad N_{\lambda^{t} \mu^{t}}^{\rho^{t}}(n-r, r)=N_{\lambda \mu}^{\rho}(r, n-r)
$$

The rim-hook formula [4] gives

$$
N_{\lambda \mu}^{\nu}(r, n-r)=\sum \epsilon(\rho / \nu) N_{\lambda \mu}^{\rho}
$$

where $\rho$ ranges over all diagrams of height $\leq r$ that can be obtained by adding $m$ rim-hooks $R_{1}, \ldots, R_{m}$, and $\epsilon\left(\rho^{t} / \nu^{t}\right)=(-1)^{\sum\left(r-\operatorname{height}\left(R_{i}\right)\right)}$.

If $\mu=(1,1, \ldots, 1)$ then

$$
V_{\mu} \otimes V_{\lambda}=V_{\rho}, \quad \rho=\left(\lambda_{1}+1, \ldots, \lambda_{r}+1\right)
$$

If $\lambda_{1}<n-r$, then since the height of $\rho$ is $\leq r$, there are no rim $n$-hooks in $\rho$. On the other hand, if $\lambda_{1}=n-r$, then it is easy to see that there is a unique rim $n$-hook in $\rho$, whose complement is $\lambda^{\prime}$ above.

We have learned from A. Postnikov that formula similar to (21) holds for the full flag variety [27]. A deeper reason for the appearance of symmetry is given by Seidel [29]. This symmetry simplifies the computation of many GromovWitten invariants. For sufficiently small $n$ and $l$ all Gromov-Witten invariants are equivalent to degree 0 ones. 


\section{Verlinde algebras}

Finally we want to explain the representation-theoretic interpretation of $\Delta_{q}(l)$ in terms of the Verlinde algebra of $S U(n)$. Denote by $\Lambda_{N}^{*}$ the set of dominant weights of $S U(n)$ at level $N$ :

$$
\Lambda_{N}^{*}=\left\{\left(\lambda_{1}, \ldots, \lambda_{n}\right) \in(\mathbb{Z} / n)^{n} \mid \lambda_{i}-\lambda_{i+1} \in \mathbb{Z}_{\geq 0}, \quad \lambda_{1}-\lambda_{n} \leq N\right\} .
$$

The Verlinde algebra $R\left(S U(n)_{N}\right)$ is the free group $\mathbb{Z}\left[\Lambda_{N}^{*}\right]$ on the generators $V_{\xi}, \xi \in \Lambda_{N}^{*}$. The algebra structure is given by "fusion product"

$$
V_{\xi_{1}} \circledast_{N} \ldots \circledast_{N} \mathcal{V}_{\xi_{l}}=\sum_{\nu \in \Lambda_{N}^{*}} m^{N}\left(\xi_{1}, \ldots, \xi_{l}, \nu\right) V_{* \nu}
$$

where the coefficients $m^{N}\left(\xi_{1}, \ldots, \xi_{l}\right)$ are defined as follows. There is a positive line bundle $L^{N}\left(\xi_{1}, \ldots, \xi_{l}\right) \rightarrow \mathcal{M}\left(\xi_{1} / N, \ldots, \xi_{l} / N\right)$ which descends from the polarizing line bundle on $\tilde{R}$ (see Pauly [26, Section 3$]$ ). The coefficient $m^{N}\left(\xi_{1}, \ldots, \xi_{l}\right)$ is defined by

$$
m^{N}\left(\xi_{1}, \ldots, \xi_{l}\right)=\operatorname{dim}\left(H^{0}\left(L^{N}\left(\xi_{1}, \ldots, \xi_{l}\right)\right) .\right.
$$

Since $L^{N}$ is positive, $\mathcal{M}\left(\xi_{1} / N, \ldots, \xi_{l} / N\right)$ is non-empty if and only if for some $k$

$$
\operatorname{dim}\left(H^{0}\left(L^{k}\left(\xi_{1}, \ldots, \xi_{l}\right)^{\otimes N}\right)=\operatorname{dim}\left(H^{0}\left(L^{k N}\left(k \xi_{1}, \ldots, k \xi_{l}\right)\right) \neq 0,\right.\right.
$$

that is, $m^{k N}\left(k \xi_{1}, \ldots, k \xi_{l}\right) \neq 0$.

Question: Does the quantum saturation conjecture hold? That is, is it true that the number $m^{N}\left(\xi_{1}, \ldots, \xi_{l}\right)$ is non-zero (and therefore positive) if and only if the inequalities in Theorem 3.1 are satisfied. This would imply that the vanishing of $m^{N}\left(\xi_{1}, \ldots, \xi_{l}\right)$ is also determined by a recursive algorithm.

\section{References}

[1] S. Agnihotri, Quantum Cohomology and the Verlinde Algebra. PhD thesis, Oxford University, 1995.

[2] P. Belkale, Local systems on $P^{1}-S$ for $S$ a finite set, University of Chicago preprint, 1998.

[3] A. Bertram, Quantum Schubert calculus, Adv. Math., 128 (1997), 289-305.

[4] A. Bertram, I. Ciocan-Fontanine, and W. Fulton, Quantum multiplication of Schur polynomials, Technical report, 1997. Available as alg-geom/9705024.

[5] I. Biswas, On the existence of unitary flat connections over the punctured sphere with given local monodromy around the punctures, Preprint, Tata Institute.

[6] A criterion for the existence of a parabolic stable bundle of rank two over the projective line, Internat. J. Math. 9 (1998), 523-533.

[7] H. Boden, Representations of orbifold groups and parabolic bundles, Comment. Math. Helv. 66 (1991), 389-447.

[8] H. Boden and K. Yokogawa, Rationality of moduli spaces of parabolic bundles, Technical report, 1996. Available as alg-geom/9610013.

[9] S. K. Donaldson and P. Kronheimer, The geometry of four-manifolds, Oxford Mathematical Monographs, Oxford University Press, New York, 1990.

[10] P. Griffiths and J. Harris, Principles of Algebraic Geometry, Wiley, New York, 1978. 
[11] V. Guillemin and E. Prato, Heckman, Kostant, and Steinberg formulas for symplectic manifolds, Adv. Math. 82 (1990), 160-179.

[12] R. Hartshorne, Algebraic Geometry, Graduate Texts in Mathematics 52, Springer-Verlag, Berlin-Heidelberg-New York, 1977.

[13] G.J. Heckman, Projections of orbits and asymptotic behavior of multiplicities for compact Lie groups, Invent. Math. 67 (1982), 333-356.

[14] U. Helmke and J. Rosenthal, Eigenvalue inequalities and Schubert calculus, Math. Nachr. 171 (1995), 207-225.

[15] L.C. Jeffrey and J. Weitsman, Bohr-Sommerfeld orbits in the moduli space of flat connections and the Verlinde dimension formula, Comm. Math. Phys. 150 (1992), 593-630.

[16] _ Symplectic geometry of the moduli space of flat connections on a Riemann surface: inductive decompositions and vanishing theorems, preprint, McGill, Santa Cruz, 1996.

[17] F.C. Kirwan, Cohomology of Quotients in Symplectic and Algebraic Geometry, Mathematical Notes 31, Princeton University Press, Princeton, 1984.

[18] A. Klyachko, Stable bundles, representation theory, and Hermitian operators, technical report.

[19] A. Knutson and T. Tao, Apiary views of the Berenstein-Zelevinsky polytope, and Klyachko's saturation conjecture, Technical report, M.I.T., 1998. e-print: math.RT/9807160.

[20] E. Lerman, E. Meinrenken, S. Tolman, and C. Woodward, Non-abelian convexity by symplectic cuts, Topology 37 (1998), 245-259.

[21] V.B. Mehta and C.S. Seshadri, Moduli of vector bundles on curves with parabolic structure, Math. Ann. 248 (1980), 205-239.

[22] E. Meinrenken and C. Woodward, Moduli spaces of flat connections on 2-manifolds, cobordism, and Witten's volume formulas, Progress in Geometry, to appear.

[23] _ Fusion of Hamiltonian loop group manifolds and cobordism, Math. Zeit., 1997, to appear.

[24] _ Hamiltonian loop group actions and Verlinde factorization, J. Diff. Geom., 1997, to appear.

[25] D. Mumford, J. Fogarty, and F. Kirwan, Geometric Invariant Theory, Ergebnisse der Mathematik und ihrer Grenzgebiete, 2. Folge 34, Springer-Verlag, Berlin-Heidelberg-New York, third edition, 1994.

[26] C. Pauly, Espaces de modules de fibrés paraboliques et blocs conformes, Duke Math. J., 84 (1996), 217-235.

[27] A. Postnikov, Hidden symmetry of Gromov-Witten invariants. In preparation.

[28] M.S. Ravi, Interpolation theory and quantum cohomology, preprint, East Carolina University.

[29] P. Seidel, $\pi_{1}$ of symplectic automorphism groups and invertibles in quantum homology rings, Geom. Funct. Anal. 7 (1997), 1046-1095.

[30] R. Sjamaar, Convexity properties of the moment mapping re-examined, Adv. Math. 138 (1998), 46-91.

[31] A. Szenes. Iterated residues and multiple Bernoulli polynomials, Internat. Math. Res. Notices 18 (1998), 937-956.

[32] H. Weyl, Das asymptotische Verteilungsgesetz der Eigenwerte linearer partieller Differentialgleichungen, Math. Ann. 71 (1912), 441-479.

[33] E. Witten, Two-dimensional gauge theories revisited, J. Geom. Phys. 9 (1992), 303-368. 
University of Amsterdam, Faculty of Mathematics, Plantage Muidergracht 24, NL-1018 TV, Amsterdam, THE NETHERLANDS

E-mail address: sharad@wins.uva.nl

Mathematics-Hill Center, Rutgers University, 110 Frelinghuysen Road, PiscatAWAY NJ 08854-8019

E-mail address: ctw@math.rutgers.edu 